\title{
Pseudocyclic Form of 4-hydroxypyrrolidine-2-carboxanilide Podands with Trioxyethylene Chain: Modeling, Conformational Search and NMR Analysis
}

Olga Borodina, ${ }^{1}$ Irina Ovchinnikova, ${ }^{2}$ Gennady Makarov, ${ }^{1}$ Oleg Yeltsov, ${ }^{3}$ Yulia Titova, ${ }^{2,3}$ Olga Fedorova, ${ }^{2}$ Artëm E. Masunov, ${ }^{4,5,1}$ Ekaterina Bartashevich ${ }^{1 *}$

${ }^{1}$ South Ural State University, 76 Lenina Avenue, Chelyabinsk 454080, Russia;

${ }^{2}$ Postovsky Institute of Organic Synthesis, Ural Branch of the Russian Academy of Sciences, 22

Kovalevskoy / 20 Akademicheskaya Street, Yekaterinburg 620108, Russia;

${ }^{3}$ Ural Federal University, 19 Mira Street, Yekaterinburg, 620002, Russia;

${ }^{4}$ NanoScience Technology Center, University of Central Florida, 12424 Research Parkway, Orlando, FL 32826, USA;

${ }^{5}$ School of Modeling, Simulation, and Training, University of Central Florida, 3100 Technology Parkway, Orlando, FL 32816, USA.

* - Corresponding author: bartashevichev@susu.ru

\section{TABLE OF CONTENTS}

Figure S1. Conformer structures of $\mathbf{2 c}$

Figure S2. 2D Spectrum of ${ }^{1} \mathrm{H}-{ }^{1} \mathrm{H}$ NOESY of the salt form of podand 1c

Figure S3. 2D Spectrum of ${ }^{1} \mathrm{H}-{ }^{1} \mathrm{H}$ NOESY of the salt form of podand 2c

Figure S4. Temperature vs chemical shift plot for the $\mathrm{H}_{\mathrm{a}}, \mathrm{H}_{\mathrm{h}}, \mathrm{H}_{\mathrm{p}}$ protons in $\mathrm{DMSOd}_{6}$ for compound 1c, $\mathbf{2 c}$

Table S1. Calculated values of proton chemical shifts of $\mathbf{2 c}$ conformers

Figure S5. The final conformer of $\mathbf{2 c}(\mathrm{U} 10+171)$

Figure S6. Variants of the arrangement of trifluoroacetate anions relative to $\mathbf{2 c}$ and the corresponding correlation diagrams 


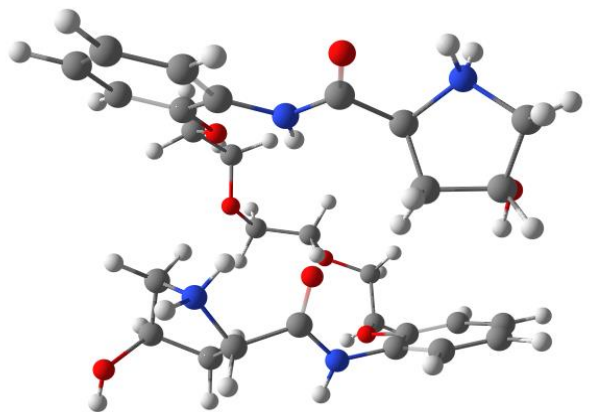

a) $\mathrm{Za} 1+0$

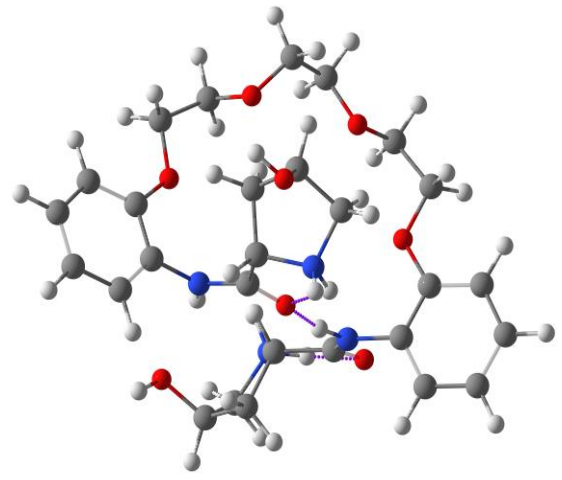

c) $\mathrm{Za} 0+128$

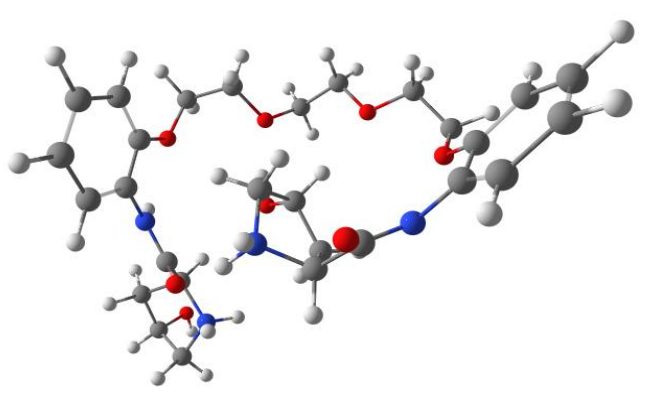

e) $\mathrm{Pa} 0-137$

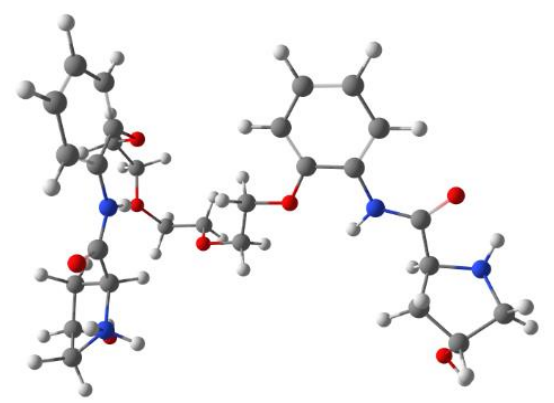

g) $\mathrm{Mb} 0-143$

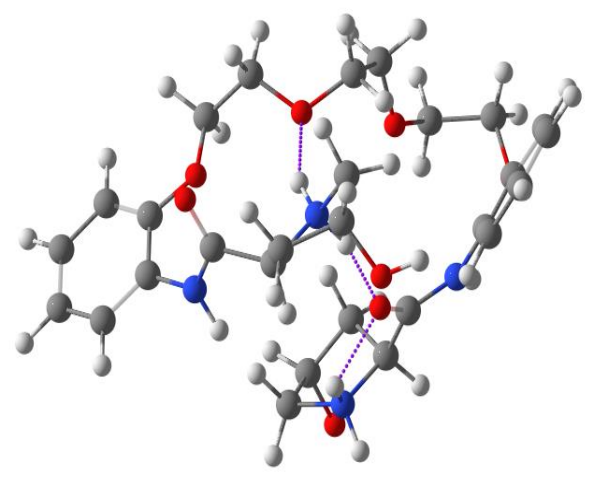

b) $\mathrm{Za} 1+115$

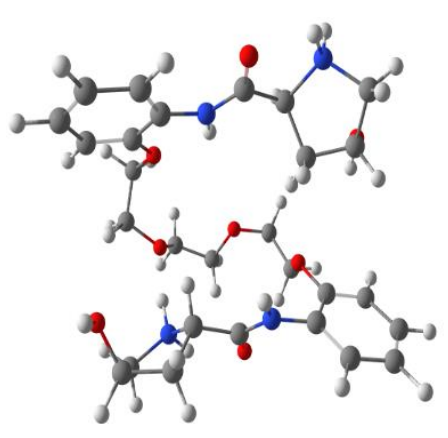

d) $\mathrm{Sa} 1+133$

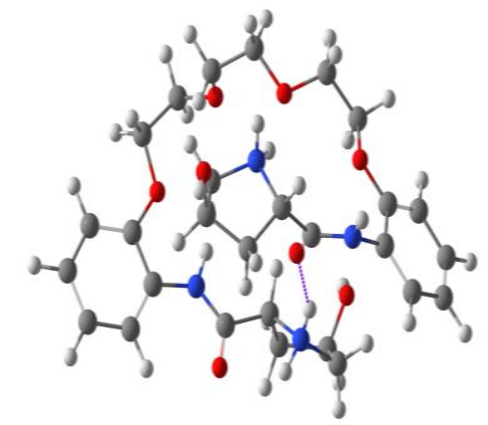

f) $\mathrm{Sa} 2+141$

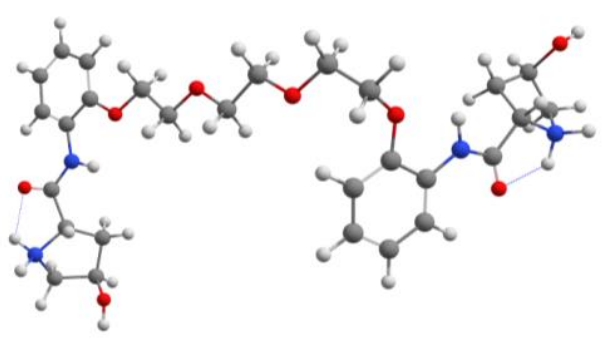

h) L10-143 


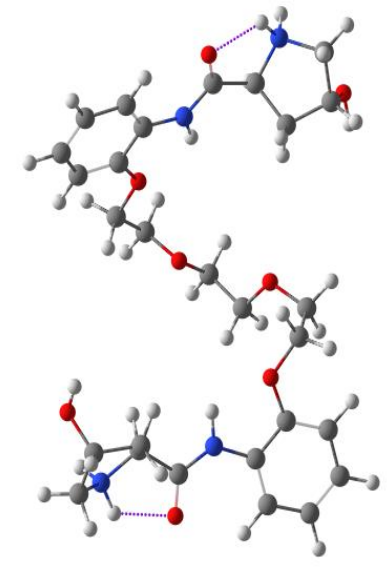

i) $\mathrm{Za} 0-143$

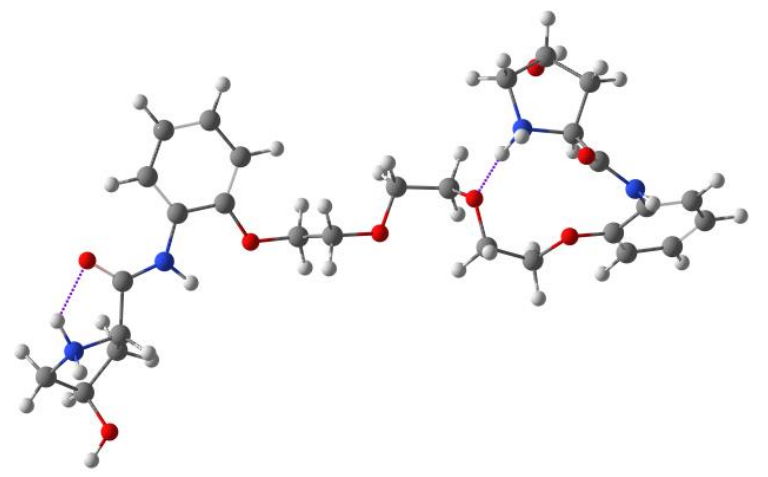

k) Pl1-144

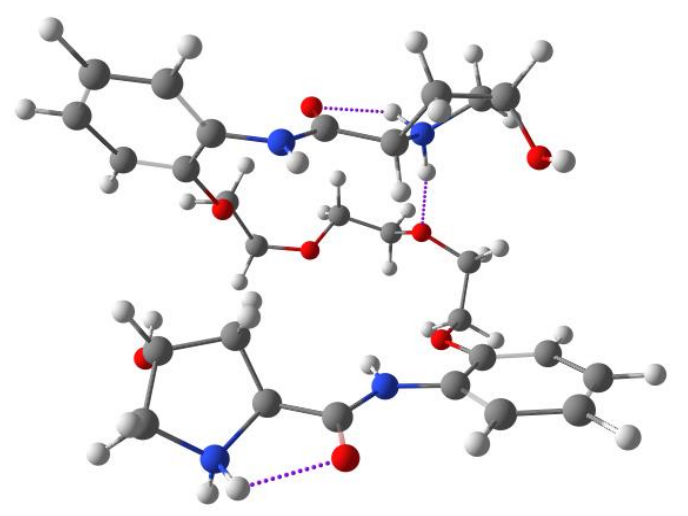

m) Sb2-146.7

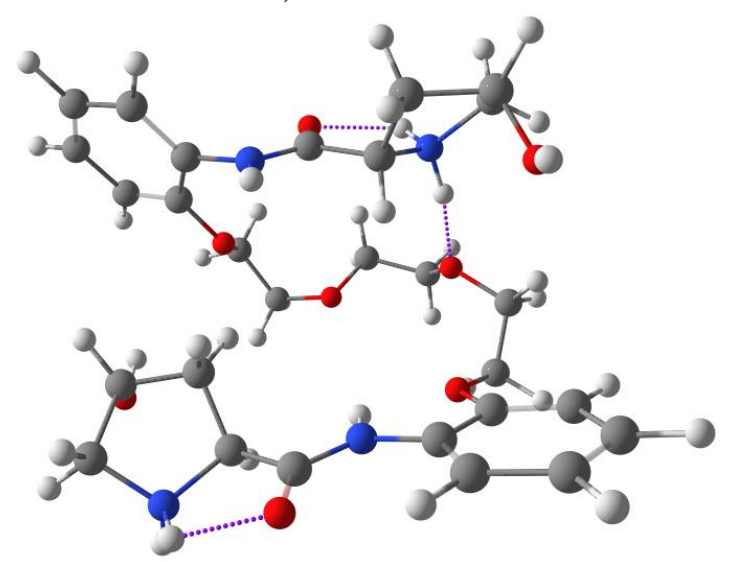

o) $\mathrm{Sb} 2-148$

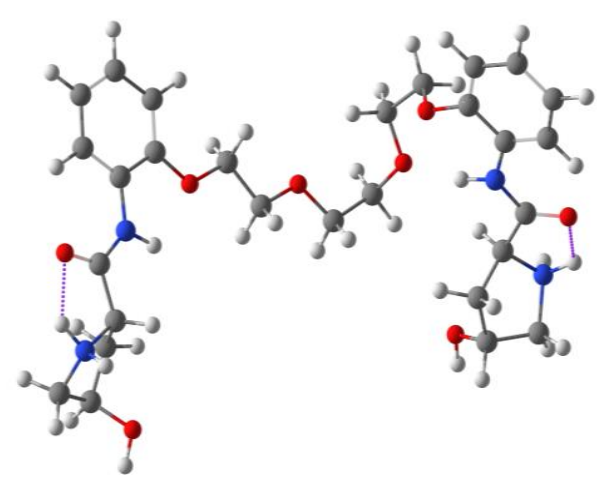

j) $\mathrm{Ma} 1-144$

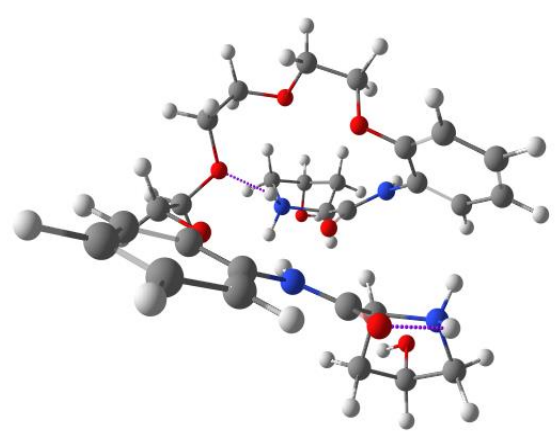

1) $\mathrm{Sb} 1+146$

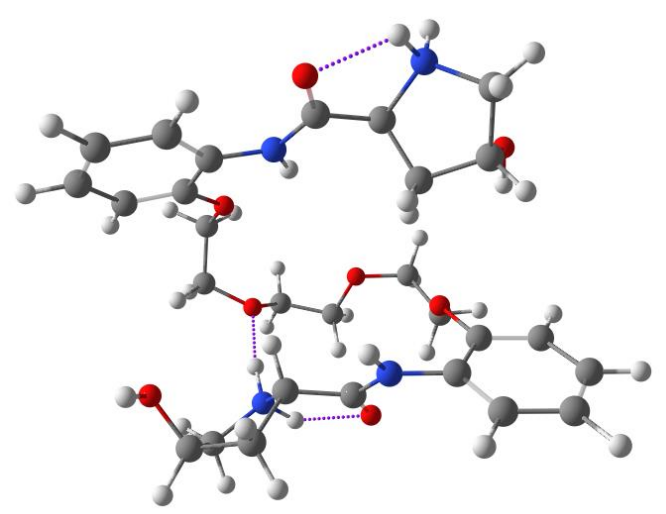

n) $\mathrm{Sb} 2-147.1$

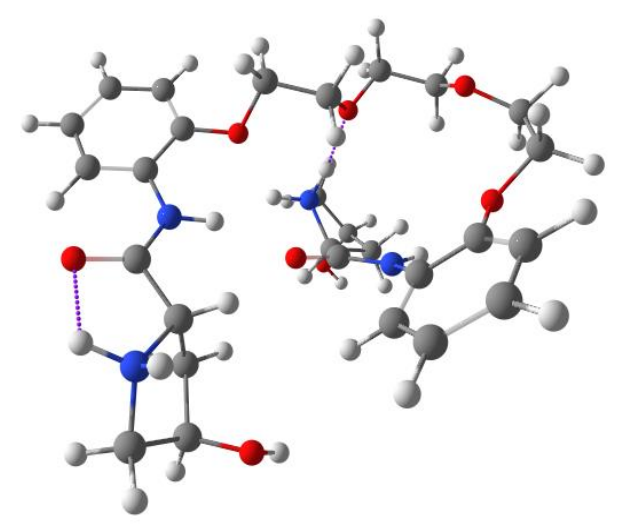

p) $\mathrm{Pa} 1+150$ 


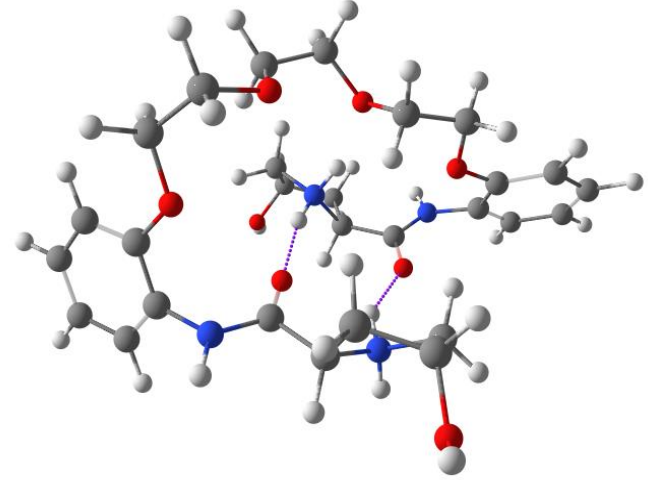

q) $\mathrm{Za} 2+152$

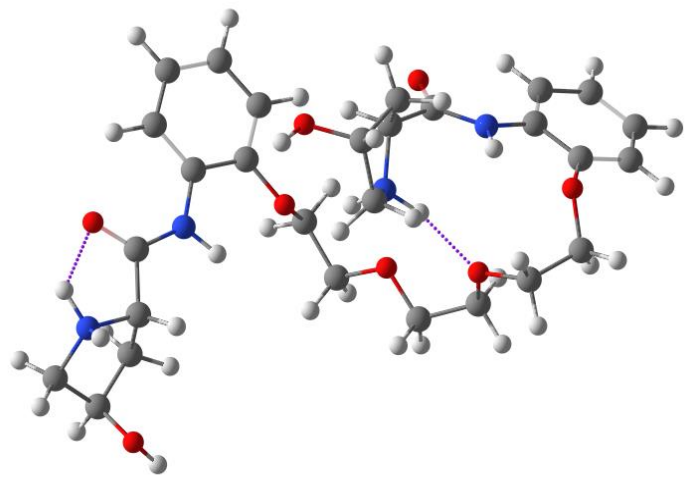

s) Pa1-163

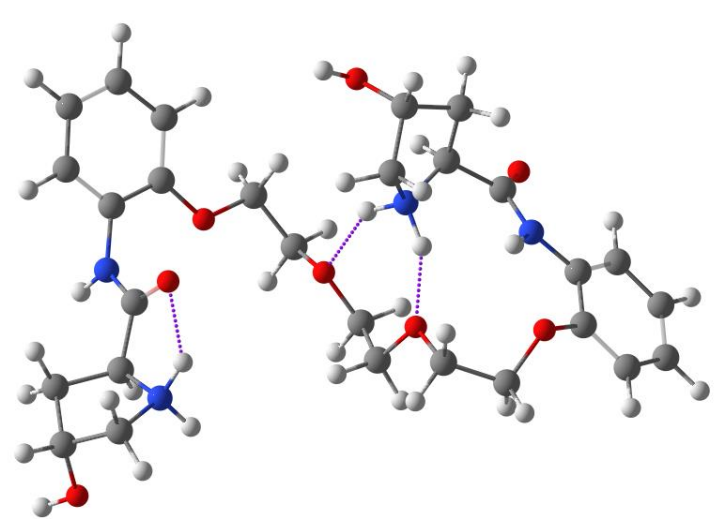

u) P11-167

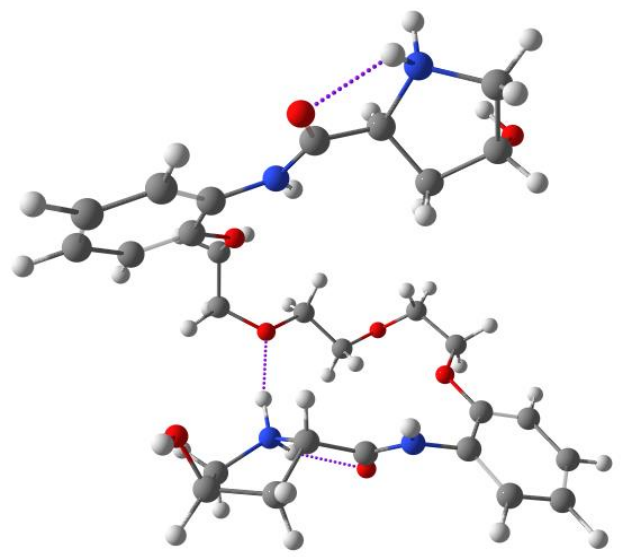

w) $\mathrm{Sa} 1-174$

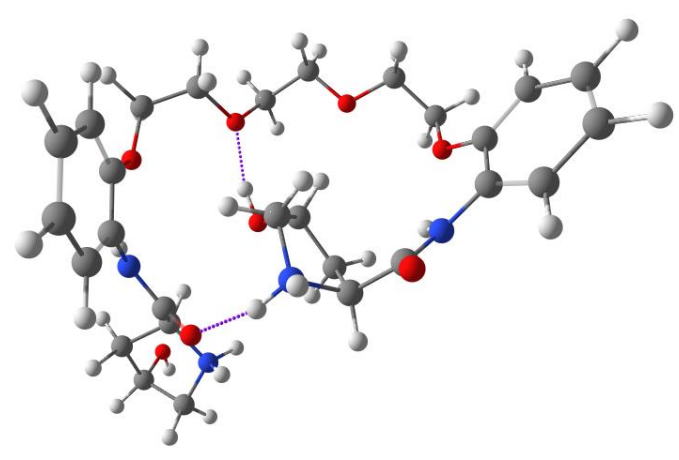

r) $\mathrm{Pa} 1+161$

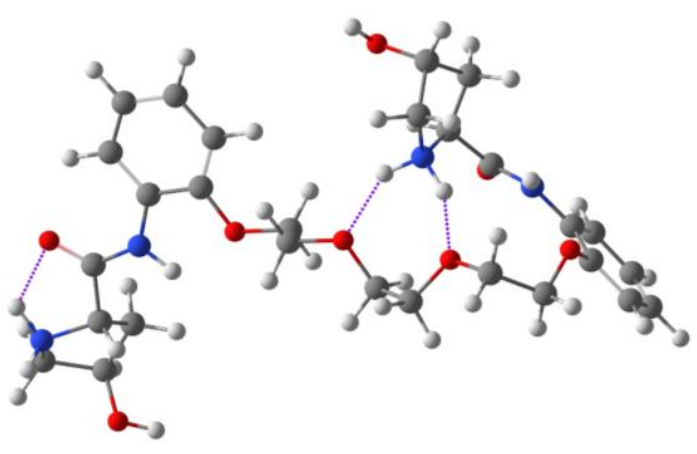

t) P11-163

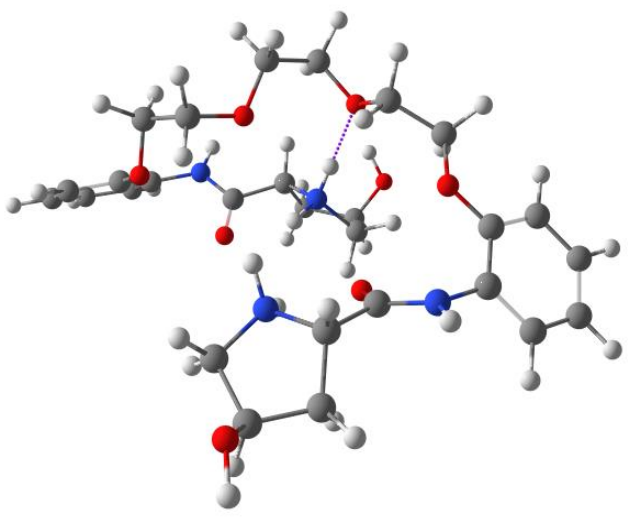

v) $\mathrm{Sa} 1-168$

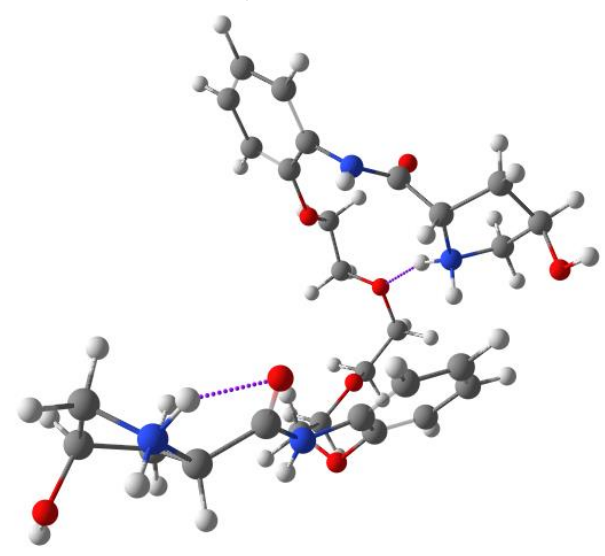

x) $\mathrm{Sa} 1-176$ 


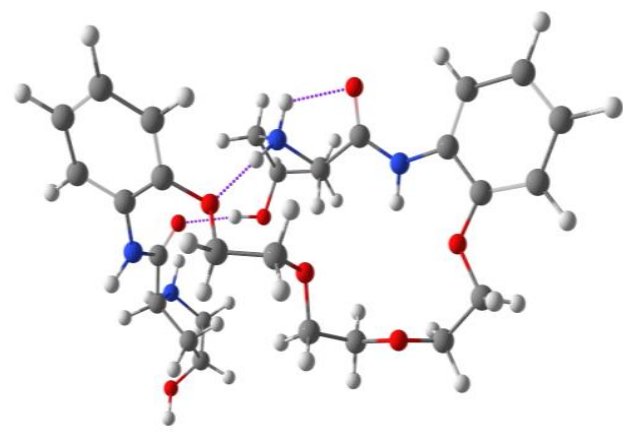

y) $\mathrm{Pa} 1+177$

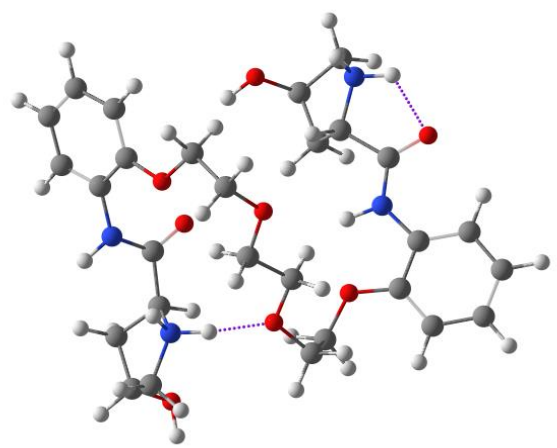

aa) Sa1-182

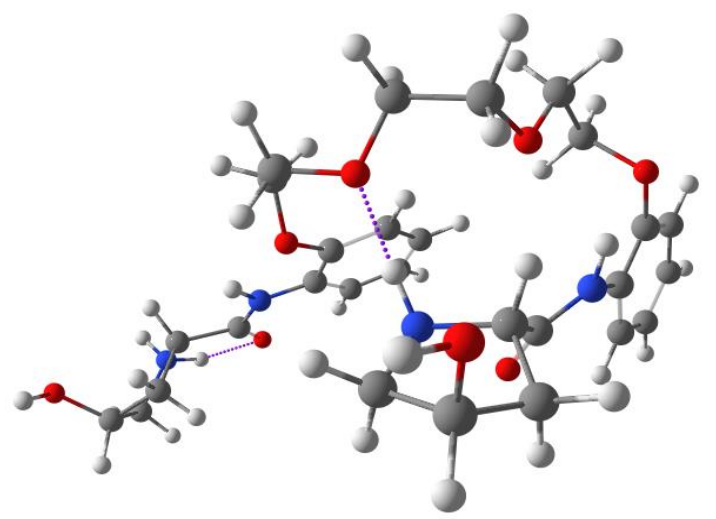

ac) $\mathrm{Pa} 1-190$

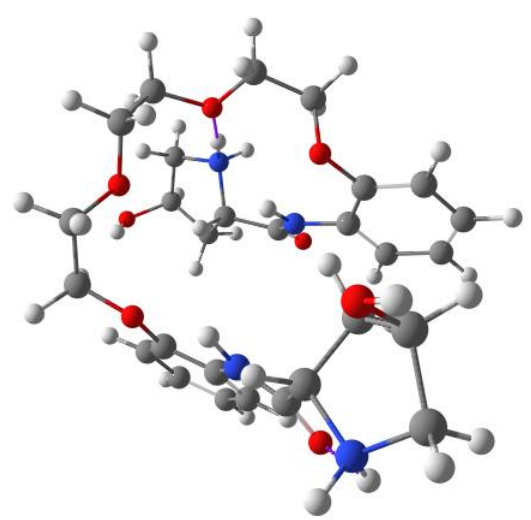

z) Zb1-177

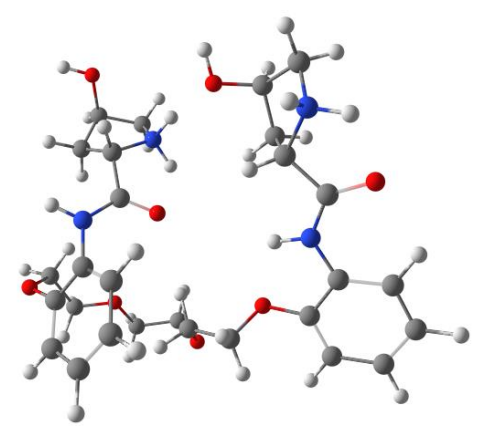

ab) $\mathrm{Ua} 0+182$

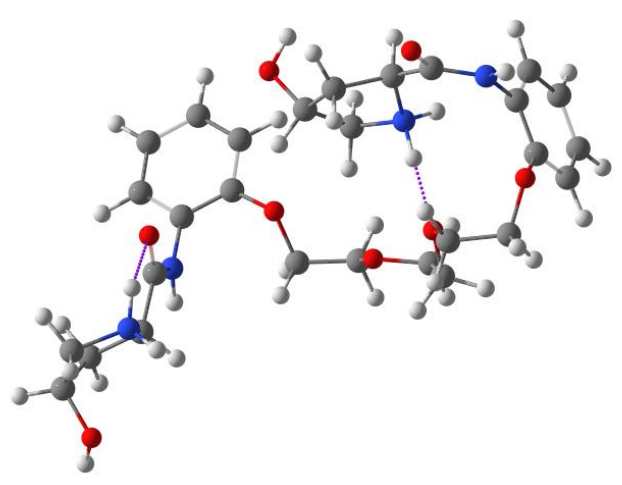

ad) P11-223

Figure S1. Conformers structures of $\mathbf{2 c}$. 


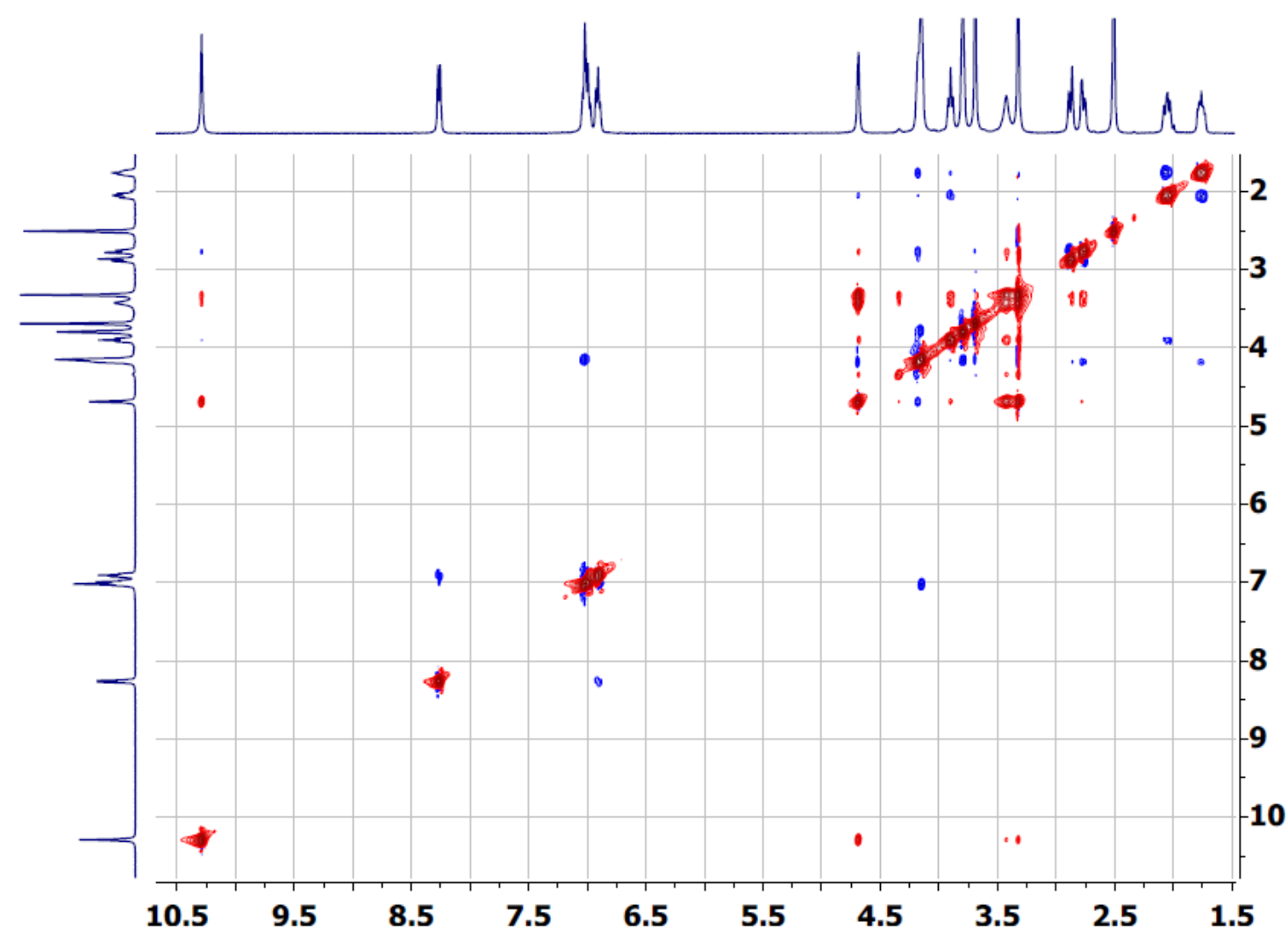

Figure S2. 2D Spectrum of ${ }^{1} \mathrm{H}-{ }^{1} \mathrm{H}$ NOESY of the podand 1c.

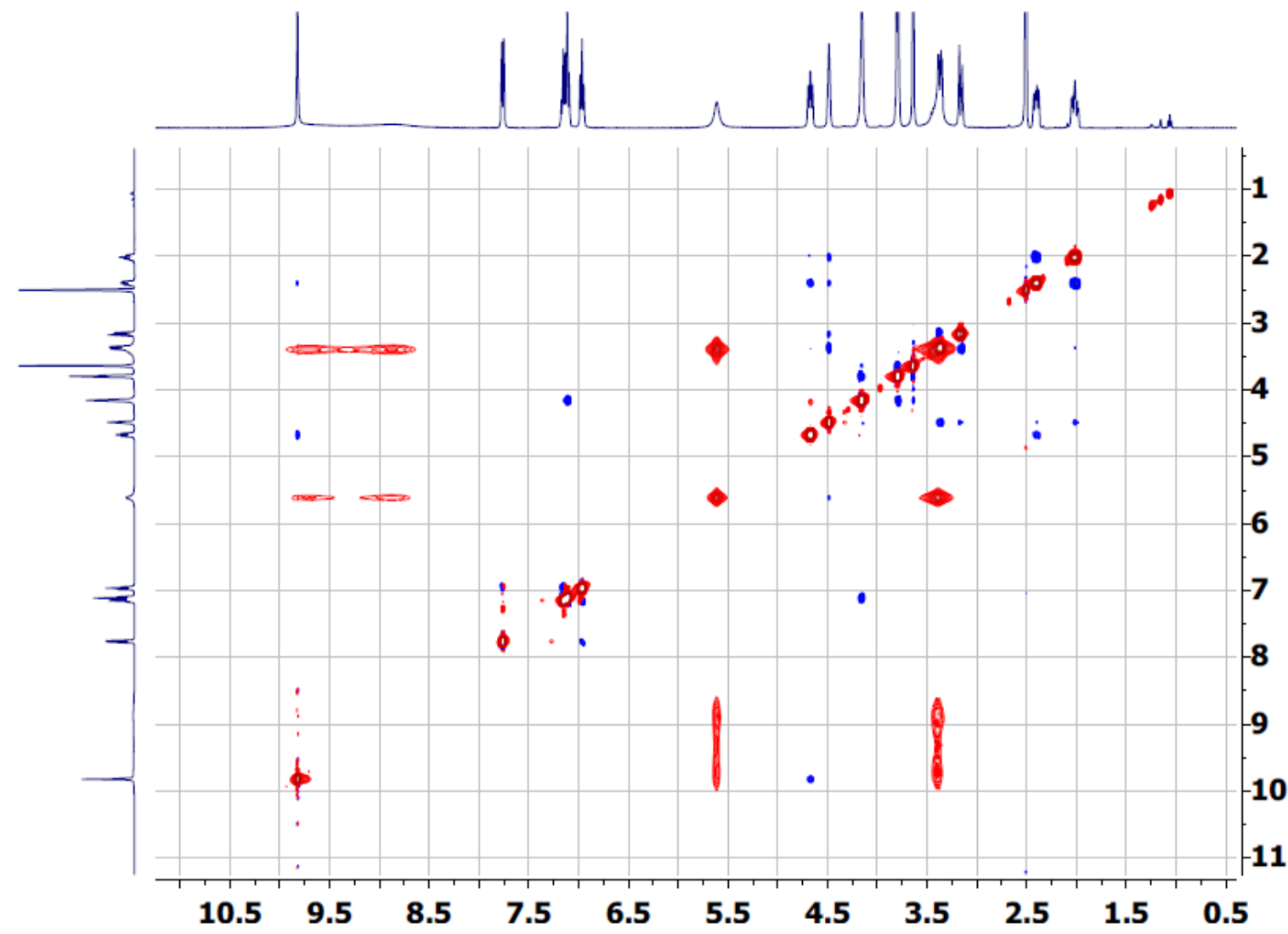

Figure S3. 2D Spectrum of ${ }^{1} \mathrm{H}-{ }^{1} \mathrm{H}$ NOESY of the salt podand 2c. 


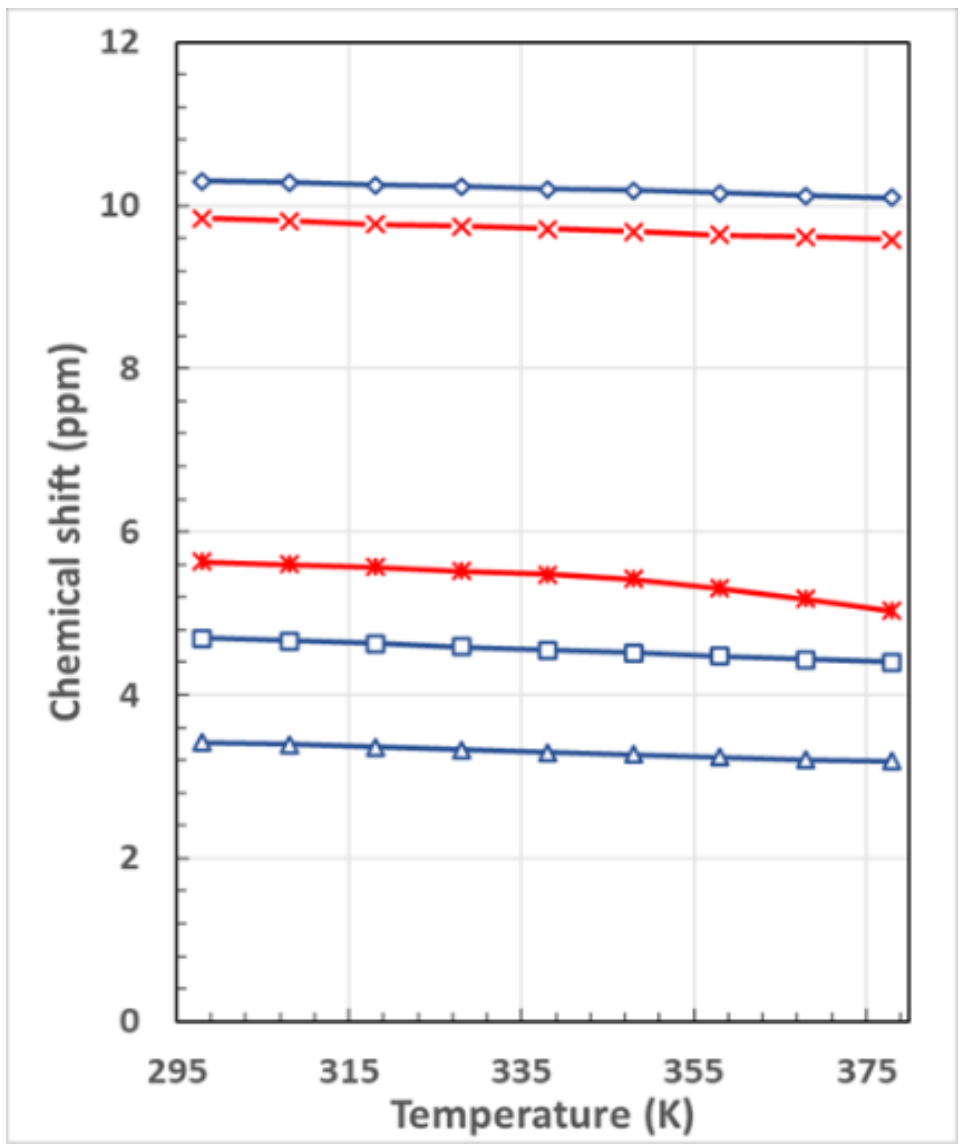

Figure S4. Temperature vs chemical shift plot for the $H_{a}(\diamond)$ or $(x), H_{h}(\square)$ or $(*), H_{p}(\Delta)$ protons in $\mathrm{DMSOd}_{6}$ for compound 1c (blue line), $2 \mathrm{c}$ (red line).

Table S1. Calculated values of proton chemical shifts of $\mathbf{2 c}$ conformers

\begin{tabular}{|c|c|c|c|c|c|c|c|c|c|c|c|c|c|c|c|}
\hline Conf. & $\begin{array}{c}\mathrm{Za} 1 \\
+0\end{array}$ & $\begin{array}{c}\mathrm{Za} 1 \\
+115\end{array}$ & $\begin{array}{c}\mathrm{Za} 0 \\
+128\end{array}$ & $\begin{array}{c}\mathrm{Sa} 1 \\
+133\end{array}$ & $\begin{array}{c}\mathrm{Pa} 0 \\
-137\end{array}$ & $\begin{array}{c}\mathrm{Sa} 2 \\
+141\end{array}$ & $\begin{array}{c}\mathrm{Mb} 0 \\
-143\end{array}$ & $\begin{array}{c}\mathrm{L} 10- \\
143\end{array}$ & $\begin{array}{c}\mathrm{Za} 0 \\
-143\end{array}$ & $\begin{array}{c}\mathrm{Ma} 1 \\
-144\end{array}$ & $\begin{array}{c}\text { P11 } \\
-144\end{array}$ & $\begin{array}{c}\text { Sb1 } \\
+146\end{array}$ & $\begin{array}{c}\text { Sb2- } \\
146.7\end{array}$ & $\begin{array}{c}\text { Sb2- } \\
147.1\end{array}$ & $\begin{array}{c}\text { Sb2 } \\
-148\end{array}$ \\
\hline \multicolumn{10}{|c|}{ branch 1} \\
\hline $\mathrm{H}_{\mathrm{a}}$ & 6.61 & 7.01 & 6.94 & 6.75 & 8.31 & 8.43 & 9.72 & 8.16 & 8.30 & 7.95 & 6.89 & 8.30 & 8.34 & 8.32 & 8.38 \\
\hline $\mathrm{H}_{\mathrm{p}}$ & 5.04 & 8.37 & 4.97 & 6.02 & 5.30 & 8.43 & 5.19 & 5.32 & 5.36 & 5.32 & 9.74 & 4.02 & 5.23 & 5.26 & 5.27 \\
\hline $\mathrm{H}_{\mathrm{p}}{ }^{\prime}$ & 7.20 & 7.25 & 7.03 & 6.10 & 7.78 & 5.82 & 7.58 & 7.84 & 7.70 & 7.89 & 6.03 & 6.64 & 7.26 & 7.40 & 7.40 \\
\hline $\mathrm{H}_{\mathrm{h}}$ & 1.42 & 1.64 & 0.93 & 1.34 & 1.44 & 1.01 & 1.46 & 1.53 & 1.37 & 1.55 & 0.97 & 1.08 & 1.34 & 1.38 & 1.25 \\
\hline $\mathrm{H}_{\mathrm{Ph}}{ }^{3}$ & 7.25 & 7.03 & 7.20 & 7.15 & 6.89 & 7.06 & 7.63 & 7.54 & 7.00 & 7.15 & 7.15 & 7.06 & 6.99 & 7.02 & 7.00 \\
\hline $\mathrm{H}_{\mathrm{Ph}}{ }^{4}$ & 7.70 & 7.60 & 7.79 & 7.79 & 7.37 & 7.41 & 7.50 & 7.36 & 7.44 & 7.50 & 7.60 & 7.43 & 7.43 & 7.42 & 7.43 \\
\hline $\mathrm{H}_{\mathrm{Ph}}{ }^{5}$ & 7.17 & 7.23 & 7.42 & 7.23 & 7.31 & 7.26 & 7.50 & 7.21 & 7.33 & 7.33 & 7.22 & 7.30 & 7.22 & 7.25 & 7.22 \\
\hline $\mathrm{H}_{\mathrm{Ph}}{ }^{6}$ & 7.54 & 7.48 & 7.50 & 7.38 & 8.73 & 8.60 & 8.86 & 8.63 & 8.79 & 8.86 & 7.56 & 8.78 & 8.72 & 8.71 & 8.72 \\
\hline $\mathrm{H}_{\mathrm{p}}{ }^{2}$ & 4.79 & 4.47 & 4.62 & 5.02 & 4.87 & 4.36 & 5.75 & 4.90 & 4.80 & 4.88 & 4.74 & 5.38 & 4.73 & 4.78 & 4.75 \\
\hline $\mathrm{H}_{\mathrm{p}}{ }^{3}$ & 2.71 & 2.44 & 1.55 & 2.49 & 2.81 & 2.24 & 2.80 & 2.71 & 2.65 & 2.69 & 2.12 & 2.17 & 3.09 & 3.21 & 3.01 \\
\hline $\mathrm{H}_{\mathrm{p}}{ }^{31}$ & 2.46 & 2.74 & 2.76 & 2.29 & 2.14 & 2.02 & 2.14 & 2.07 & 2.20 & 2.06 & 2.03 & 1.88 & 1.86 & 1.78 & 1.85 \\
\hline $\mathrm{H}_{\mathrm{p}}{ }^{4}$ & 4.90 & 4.92 & 4.64 & 4.86 & 4.88 & 4.59 & 4.89 & 4.74 & 4.94 & 4.85 & 4.77 & 4.72 & 4.17 & 4.09 & 4.20 \\
\hline $\mathrm{H}_{\mathrm{p}}{ }^{51}$ & 4.54 & 3.09 & 3.15 & 3.37 & 3.54 & 3.21 & 3.59 & 3.49 & 3.54 & 3.47 & 3.56 & 3.39 & 3.51 & 3.42 & 3.45 \\
\hline $\mathrm{H}_{\mathrm{p}}{ }^{5}$ & 3.19 & 3.18 & 3.38 & 3.30 & 3.52 & 2.11 & 3.73 & 3.51 & 3.79 & 3.55 & 3.42 & 3.43 & 3.64 & 3.67 & 3.64 \\
\hline \multicolumn{10}{|c|}{ branch 2 } \\
\hline $\mathrm{H}_{\mathrm{a}}$ & 8.28 & 8.34 & 9.64 & 6.57 & 8.31 & 7.29 & 7.96 & 8.13 & 8.22 & 9.41 & 8.00 & 7.04 & 6.60 & 6.49 & 6.62 \\
\hline
\end{tabular}




\begin{tabular}{|c|c|c|c|c|c|c|c|c|c|c|c|c|c|c|c|}
\hline $\mathrm{H}_{\mathrm{p}}$ & 10 & .51 & 5.25 & 6.40 & 5.37 & 5.40 & 5.26 & 5.34 & 5.27 & 5.22 & 5.26 & 5.58 & 9.65 & 9.73 & 9.67 \\
\hline $\mathrm{H}_{\mathrm{p}}{ }^{\prime}$ & .19 & .30 & 7.96 & 6.54 & 7.84 & 7.26 & 7.64 & 7.76 & 7.79 & 7.56 & 7.78 & 8.51 & 6.74 & 6.69 & 6.89 \\
\hline $\mathrm{H}_{\mathrm{h}}$ & 81 & 43 & .38 & .33 & 1.47 & 1.35 & 1.45 & 1.50 & 1.30 & 1.43 & 1.57 & .47 & 0.77 & 0.88 & 0.73 \\
\hline $\mathrm{H}_{\mathrm{Ph}}{ }^{3}$ & 01 & 47 & .16 & 7.59 & 7.08 & 7.19 & 6.62 & 7.12 & 7.04 & 7.58 & 7.08 & 7.51 & 7.54 & 7.52 & 7.55 \\
\hline $\mathrm{H}_{\mathrm{Ph}^{4}}$ & 7.43 & 7.64 & 7.78 & 7.77 & 7.44 & 7.82 & 7.18 & 7.35 & 7.43 & 7.36 & 7.39 & 7.81 & 7.67 & 7.67 & 7.66 \\
\hline $\mathrm{H}_{\mathrm{Ph}}{ }^{5}$ & 7.33 & 7.35 & 7.31 & 7.48 & 7.24 & 7.35 & 7.19 & 7.06 & 7.23 & 7.56 & 7.31 & 7.58 & 7.45 & 7.40 & 7.40 \\
\hline $\mathrm{H}_{\mathrm{Ph}}{ }^{6}$ & 8.63 & 8.01 & 7.46 & 7.58 & 8.75 & 7.58 & 8.69 & 8.67 & 8.70 & 8.69 & 8.83 & 7.59 & 7.33 & 7.32 & 7.35 \\
\hline $\mathrm{H}_{\mathrm{p}}^{2}$ & 4.49 & 4.92 & 4.91 & 5.02 & 4.85 & 4.46 & 4.77 & 4.73 & 4.71 & 4.89 & 4.85 & 4.98 & 4.37 & 4.31 & 4.38 \\
\hline $\mathrm{H}_{\mathrm{p}}{ }^{3}$ & 2.87 & 2.69 & 2.75 & 2.47 & 2.77 & 2.45 & 2.62 & 2.71 & 2.38 & 2.69 & 2.71 & 2.43 & 2.16 & 2.15 & 2.18 \\
\hline $\mathrm{H}_{\mathrm{p}}{ }^{31}$ & 1.98 & 2.15 & 2.32 & 2.22 & 2.18 & 2.14 & 1.99 & 2.12 & 2.14 & 2.15 & 2.05 & 2.52 & 2.00 & 1.98 & 1.99 \\
\hline $\mathrm{H}_{\mathrm{p}}{ }^{4}$ & 4.82 & 4.63 & 4.92 & 4.88 & 4.91 & 4.51 & 4.84 & 4.87 & 4.86 & 4.89 & 4.92 & 4.96 & 4.72 & 4.69 & 4.73 \\
\hline $\mathrm{H}_{\mathrm{p}}{ }^{5 \prime}$ & 3.70 & 3.68 & 3.66 & 3.59 & 3.54 & 3.37 & 3.50 & 3.58 & 3.75 & 3.51 & 3.56 & 3.75 & 3.46 & 3.43 & 3.47 \\
\hline $\mathrm{H}_{\mathrm{p}}{ }^{5}$ & 3.65 & 3.09 & 3.55 & 3.52 & 3.56 & 3.01 & 3.49 & 3.53 & 3.55 & 3.57 & 3.50 & 3.64 & 3.39 & 3.38 & 3.37 \\
\hline \multicolumn{16}{|c|}{ polyester chain } \\
\hline $\mathrm{OCH}_{2}$ & 62 & .06 & 4.16 & 4.76 & 4.12 & 4.25 & 4.46 & 4.37 & 4.09 & 4.27 & 3.93 & 4.60 & 4.93 & 4.91 & 4.95 \\
\hline $\mathrm{OCH}_{2}$ & 4.58 & 3.84 & 4.18 & 3.94 & 4.00 & 4.08 & 3.72 & 4.50 & 4.32 & 4.25 & 4.44 & 3.74 & 4.41 & 4.32 & 4.35 \\
\hline $\mathrm{OCH}_{2}$ & 3.78 & 47 & 4.46 & 4.28 & 3.98 & 4.51 & 3.85 & 3.86 & 3.77 & 4.02 & 4.31 & 4.48 & 4.42 & 4.45 & 4.45 \\
\hline $\mathrm{OCH}_{2}$ & 3.84 & 4.01 & 3.27 & 3.44 & 4.64 & 3.85 & 4.05 & 3.90 & 3.97 & 3.98 & 4.40 & 4.00 & 3.66 & 3.69 & 3.66 \\
\hline $\mathrm{OCH}_{2}$ & 3.52 & 3.10 & 3.55 & 4.28 & 4.09 & 3.19 & 4.16 & 3.76 & 3.46 & 4.09 & 3.81 & 3.02 & 3.83 & 3.88 & 3.86 \\
\hline $\mathrm{OCH}_{2}$ & 3.88 & 2.56 & 4.16 & 3.18 & 3.85 & 3.91 & 3.63 & 3.62 & 3.78 & 3.91 & 3.89 & 4.39 & 4.14 & 4.17 & 4.07 \\
\hline $\mathrm{OCH}_{2}$ & 3.80 & 4.21 & 4.01 & 4.13 & 4.03 & 4.23 & 4.20 & 4.14 & 4.57 & 3.70 & 4.66 & 3.92 & 4.51 & 4.58 & 4.16 \\
\hline $\mathrm{OCH}_{2}$ & 4.57 & 4.53 & 4.30 & 3.75 & 4.48 & 4.23 & 3.74 & 4.18 & 4.64 & 4.30 & 4.34 & 3.76 & 4.07 & 4.10 & 4.24 \\
\hline $\mathrm{OCH}_{2}$ & 3.66 & 3.42 & 3.63 & 3.13 & 3.79 & 4.33 & 3.83 & 3.96 & 3.89 & 5.03 & 3.69 & 3.98 & 4.51 & 4.56 & 4.45 \\
\hline $\mathrm{OCH}_{2}$ & 4.25 & 4.17 & 4.03 & 4.43 & 4.04 & 3.62 & 4.27 & 3.98 & 4.02 & 3.72 & 4.05 & 3.58 & 3.77 & 3.80 & 3.78 \\
\hline $\mathrm{OCH}_{2}$ & 3.99 & 2.90 & 3.18 & 4.25 & 3.60 & 4.02 & 4.32 & 3.83 & 3.39 & 3.97 & 4.09 & 3.53 & 3.16 & 3.22 & 3.14 \\
\hline $\mathrm{OCH}_{2}$ & 3.58 & 3.59 & 4.05 & 3.66 & 4.09 & 3.75 & 3.48 & 3.69 & 4.37 & 4.13 & 3.77 & 4.01 & 4.20 & 4.18 & 4.13 \\
\hline
\end{tabular}

\begin{tabular}{|c|c|c|c|c|c|c|c|c|c|c|c|c|c|c|c|}
\hline Conf. & $\begin{array}{c}\mathrm{Pa} 1 \\
+150 \\
\end{array}$ & $\begin{array}{c}\mathrm{Za} 2 \\
+152 \\
\end{array}$ & $\begin{array}{c}\text { Pa1 } \\
+161 \\
\end{array}$ & $\begin{array}{c}\mathrm{Pa} 1 \\
-163 \\
\end{array}$ & $\begin{array}{c}\text { Pl1 } \\
-163\end{array}$ & $\begin{array}{c}\text { P11 } \\
-167\end{array}$ & $\begin{array}{c}\text { Sa1 } \\
-168 \\
\end{array}$ & $\begin{array}{c}\text { Sa1 } \\
-174\end{array}$ & $\begin{array}{c}\text { Sa1 } \\
-176\end{array}$ & $\begin{array}{l}\text { Pa1 } \\
+177 \\
\end{array}$ & $\begin{array}{c}\text { Zb1 } \\
-177\end{array}$ & $\begin{array}{c}\text { Sa1 } \\
-182\end{array}$ & $\begin{array}{r}\mathrm{Ua} 0 \\
+182 \\
\end{array}$ & $\begin{array}{c}\text { Pa1 } \\
-190\end{array}$ & $\begin{array}{l}\text { P11 } \\
-223\end{array}$ \\
\hline \multicolumn{16}{|c|}{ branch 1} \\
\hline $\mathrm{H}_{\mathrm{a}}$ & .82 & 7.27 & 8.32 & 8.03 & 8.07 & 6.63 & 7.05 & 8.28 & 6.80 & 7.03 & 7.75 & 8.08 & 8.73 & 8.21 & 6.54 \\
\hline $\mathrm{H}_{\mathrm{p}}$ & 5.59 & 9.23 & 7.02 & 5.26 & 5.26 & 5.33 & 9.63 & 5.20 & 5.16 & 5.46 & 8.49 & 5.30 & 5.33 & 5.38 & 5.33 \\
\hline $\mathrm{H}_{\mathrm{p}}{ }^{\prime}$ & 9.87 & 7.36 & 5.63 & 7.63 & 7.50 & 7.73 & 6.29 & 7.72 & 8.26 & 6.80 & 4.02 & 7.84 & 7.95 & 7.62 & 7.57 \\
\hline $\mathrm{H}_{\mathrm{h}}$ & 1.39 & 1.19 & 5.21 & 1.46 & 1.26 & 1.46 & 1.15 & 1.41 & 1.56 & 1.54 & 1.14 & 1.08 & 2.09 & 1.43 & 1.38 \\
\hline $\mathrm{H}_{\mathrm{Ph}}{ }^{3}$ & 7.22 & 7.16 & 7.04 & 7.95 & 7.03 & 7.06 & 7.60 & 7.13 & 7.36 & 7.45 & 7.40 & 6.98 & 7.39 & 7.06 & 7.17 \\
\hline $\mathrm{H}_{\mathrm{Ph}}{ }^{4}$ & 7.63 & 7.74 & 7.44 & 7.60 & 7.37 & 7.74 & 7.72 & 7.40 & 7.63 & 7.87 & 7.41 & 7.45 & 7.53 & 6.92 & 7.63 \\
\hline $\mathrm{H}_{\mathrm{Ph}}{ }^{5}$ & 7.35 & 7.26 & 7.26 & 7.79 & 7.32 & 7.34 & 7.62 & 7.37 & 7.29 & 7.65 & 7.49 & 7.40 & 7.37 & 7.22 & 7.35 \\
\hline $\mathrm{H}_{\mathrm{Ph}}{ }^{6}$ & 7.89 & 7.52 & 8.02 & 8.93 & 8.81 & 7.44 & 7.41 & 8.84 & 7.32 & 7.57 & 8.11 & 8.78 & 8.63 & 8.66 & 7.35 \\
\hline $\mathrm{H}_{\mathrm{p}}^{2}$ & 4.67 & 4.59 & 4.37 & 4.78 & 4.61 & 4.86 & 4.29 & 4.52 & 3.79 & 4.80 & 3.71 & 4.76 & 4.67 & 4.73 & 4.72 \\
\hline $\mathrm{H}_{\mathrm{p}}{ }^{3}$ & 2.36 & 2.30 & 1.80 & 2.45 & 2.39 & 2.66 & 2.51 & 2.59 & 2.41 & 2.63 & 1.88 & 2.58 & 2.62 & 2.53 & 2.58 \\
\hline $\mathrm{H}_{\mathrm{p}}{ }^{3 \prime}$ & 2.26 & 2.02 & 2.88 & 2.11 & 2.11 & 2.29 & 2.36 & 2.22 & 2.29 & 2.35 & 2.94 & 2.20 & 2.33 & 2.17 & 2.24 \\
\hline $\mathrm{H}_{\mathrm{p}}{ }^{4}$ & 4.95 & 4.77 & 5.31 & 4.93 & 4.92 & 4.95 & 4.52 & 4.56 & 4.91 & 4.91 & 4.72 & 4.79 & 5.13 & 4.85 & 4.92 \\
\hline $\mathrm{H}_{\mathrm{p}}{ }^{\prime \prime}$ & 3.80 & 3.39 & 2.35 & 3.56 & 3.57 & 3.46 & 3.47 & 3.78 & 3.50 & 3.57 & 4.10 & 3.42 & 3.83 & 3.51 & 3.40 \\
\hline $\mathrm{H}_{\mathrm{p}}{ }^{5}$ & 3.37 & 3.13 & 1.83 & 3.73 & 3.78 & 3.70 & 3.33 & 3.67 & 3.62 & 3.79 & 3.17 & 3.63 & 3.71 & 3.78 & 3.71 \\
\hline \multicolumn{16}{|c|}{ branch 2} \\
\hline $\mathrm{H}_{\mathrm{a}}$ & 9.70 & 7.02 & 7.37 & 8.62 & 8.04 & 8.26 & 6.99 & 6.69 & 7.86 & 8.31 & 8.25 & 6.97 & 6.89 & 9.06 & 7.02 \\
\hline $\mathrm{H}_{\mathrm{p}}$ & 5.24 & 5.01 & 5.39 & 7.63 & 8.12 & 7.85 & 5.06 & 8.14 & 5.49 & 8.40 & 5.04 & 9.86 & 8.87 & 8.88 & 3.91 \\
\hline
\end{tabular}




\begin{tabular}{|c|c|c|c|c|c|c|c|c|c|c|c|c|c|c|c|}
\hline $\mathrm{H}_{\mathrm{p}}{ }^{\prime}$ & .37 & .57 & .18 & 7.98 & 8.65 & 8.92 & 6.63 & 5.83 & 7.78 & 7.02 & 6.98 & 4.46 & 6.81 & 5.15 & 7.08 \\
\hline $\mathrm{H}_{\mathrm{h}}$ & 1.51 & .40 & 1.70 & 1.14 & 1.71 & 1.35 & 2.01 & 0.75 & 1.51 & 2.99 & 1.58 & 1.81 & 1.44 & 1.56 & 0.84 \\
\hline $\mathrm{H}_{\mathrm{Ph}}{ }^{3}$ & 7.42 & .12 & 7.06 & 7.40 & 7.30 & 7.35 & 7.07 & 7.10 & 7.79 & 7.09 & 7.06 & 7.07 & 7.62 & 7.58 & 7.44 \\
\hline $\mathrm{H}_{\mathrm{Ph}}{ }^{4}$ & 7.51 & 7.82 & 7.50 & 7.55 & 7.49 & 7.39 & 7.74 & 7.73 & 7.51 & 7.42 & 7.45 & 7.68 & 7.59 & 7.44 & 7.74 \\
\hline $\mathrm{H}_{\mathrm{Ph}}^{5}$ & 7.44 & 7.39 & 7.41 & 7.45 & 7.40 & 7.43 & 7.27 & 7.33 & 7.55 & 7.29 & 7.21 & 7.29 & 7.20 & 7.45 & 7.45 \\
\hline $\mathrm{H}_{\mathrm{Ph}}{ }^{6}$ & 8.53 & 7.51 & 7.97 & 8.60 & 8.38 & 8.60 & 7.44 & 7.37 & 8.69 & 8.40 & 8.42 & 7.46 & 7.50 & 8.16 & 7.79 \\
\hline $\mathrm{H}_{\mathrm{p}}{ }^{2}$ & 5.49 & 4.77 & 4.90 & 4.51 & 4.51 & 4.47 & 4.75 & 4.29 & 4.85 & 5.84 & 4.64 & 4.67 & 4.84 & 4.62 & 4.53 \\
\hline $\mathrm{H}_{\mathrm{p}}{ }^{3}$ & 2.72 & 2.57 & 2.83 & 2.49 & 2.84 & 2.89 & 2.64 & 2.21 & 2.70 & 2.59 & 2.34 & 2.73 & 2.60 & 2.58 & 2.51 \\
\hline $\mathrm{H}_{\mathrm{p}}{ }^{31}$ & 2.27 & 2.44 & 2.31 & 2.18 & 2.32 & 2.28 & 2.21 & 1.97 & 2.17 & 2.10 & 1.43 & 2.22 & 2.31 & 2.19 & 2.35 \\
\hline $\mathrm{H}_{\mathrm{p}}{ }^{4}$ & 4.96 & 4.76 & 4.96 & 4.66 & 4.88 & 4.75 & 4.92 & 4.74 & 4.88 & 4.78 & 4.74 & 4.87 & 4.98 & 4.85 & 4.22 \\
\hline $\mathrm{H}_{\mathrm{p}}{ }^{51}$ & 3.82 & 3.44 & 3.67 & 3.19 & 3.54 & 3.33 & 3.62 & 3.48 & 3.68 & 3.31 & 3.48 & 3.41 & 3.53 & 3.44 & 2.83 \\
\hline $\mathrm{H}_{\mathrm{p}}{ }^{5}$ & 3.68 & 3.69 & 3.63 & 3.51 & 3.64 & 3.52 & 4.25 & 3.40 & 3.31 & 3.30 & 3.65 & 3.70 & 3.51 & 3.49 & 4.84 \\
\hline \multicolumn{16}{|c|}{ polyester chain } \\
\hline $\mathrm{OCH}_{2}$ & 4.39 & 3.87 & 4.54 & 4.78 & 4.34 & 4.28 & 4.22 & 4.94 & 4.46 & 4.71 & 3.45 & 4.56 & 3.74 & 4.40 & 3.38 \\
\hline $\mathrm{OCH}_{2}$ & 4.17 & 4.19 & 4.61 & 3.72 & 4.49 & 4.02 & 3.68 & 4.14 & 4.16 & 3.31 & 4.09 & 4.68 & 4.09 & 4.19 & 4.69 \\
\hline $\mathrm{OCH}_{2}$ & 4.18 & 54 & 3.80 & 3.86 & 4.32 & 3.88 & 3.52 & 4.63 & 4.28 & 4.41 & 4.26 & 3.9 & 2.43 & 3.69 & 3.26 \\
\hline $\mathrm{OCH}_{2}$ & 3.70 & 3.65 & 3.29 & 3.61 & 3.52 & 3.90 & 4.14 & 3.75 & 3.34 & 3.54 & 3.65 & 4.07 & & 4.46 & 3.72 \\
\hline $\mathrm{OCH}_{2}$ & 3.93 & & 3.83 & 3.91 & 3.29 & 3.40 & 3.37 & 3.44 & 3.65 & 3.39 & 3.76 & 3.58 & & 4.50 & 3.05 \\
\hline $\mathrm{OCH}_{2}$ & 3.63 & 4.06 & 3.23 & 3.31 & 4.08 & 3.91 & 3.86 & 4.24 & 4.03 & 4.12 & 4.08 & 4.33 & 3.82 & 3.43 & 2.86 \\
\hline $\mathrm{OCH}_{2}$ & 3.33 & 3.67 & 4.62 & 4.53 & 4.63 & 4.64 & 3.83 & 4.29 & 3.74 & 4.00 & 4.24 & 4.12 & 4.36 & 3.75 & 4.04 \\
\hline $\mathrm{OCH}_{2}$ & 4.51 & 4.57 & 4.60 & 4.10 & 3.96 & 3.93 & 4.84 & 4.04 & 4.44 & 4.21 & 4.71 & 4.12 & 4.33 & 4.60 & 4.96 \\
\hline $\mathrm{OCH}_{2}$ & 3.47 & 3.79 & 3.20 & 3.77 & 3.54 & 3.56 & 4.17 & 4.01 & 4.31 & 3.70 & 3.79 & 4.00 & 4.01 & 4.12 & 3.48 \\
\hline $\mathrm{OCH}_{2}$ & 4.03 & 4.43 & 4.23 & 4.38 & 4.38 & 4.38 & 3.93 & 3.96 & 3.51 & 4.28 & 4.14 & 3.75 & 3.46 & 3.83 & 4.05 \\
\hline $\mathrm{OCH}_{2}$ & 4.33 & 3.88 & 4.24 & 3.88 & 3.48 & 3.53 & 3.65 & 3.98 & 3.46 & 3.79 & 3.31 & 3.68 & 3.52 & 4.40 & 3.62 \\
\hline $\mathrm{OCH}_{2}$ & 3.92 & 3.18 & 3.76 & 3.11 & 3.90 & 3.89 & 4.08 & 3.73 & 3.90 & 3.85 & 4.30 & 4.23 & 3.31 & 3.77 & 3.43 \\
\hline
\end{tabular}

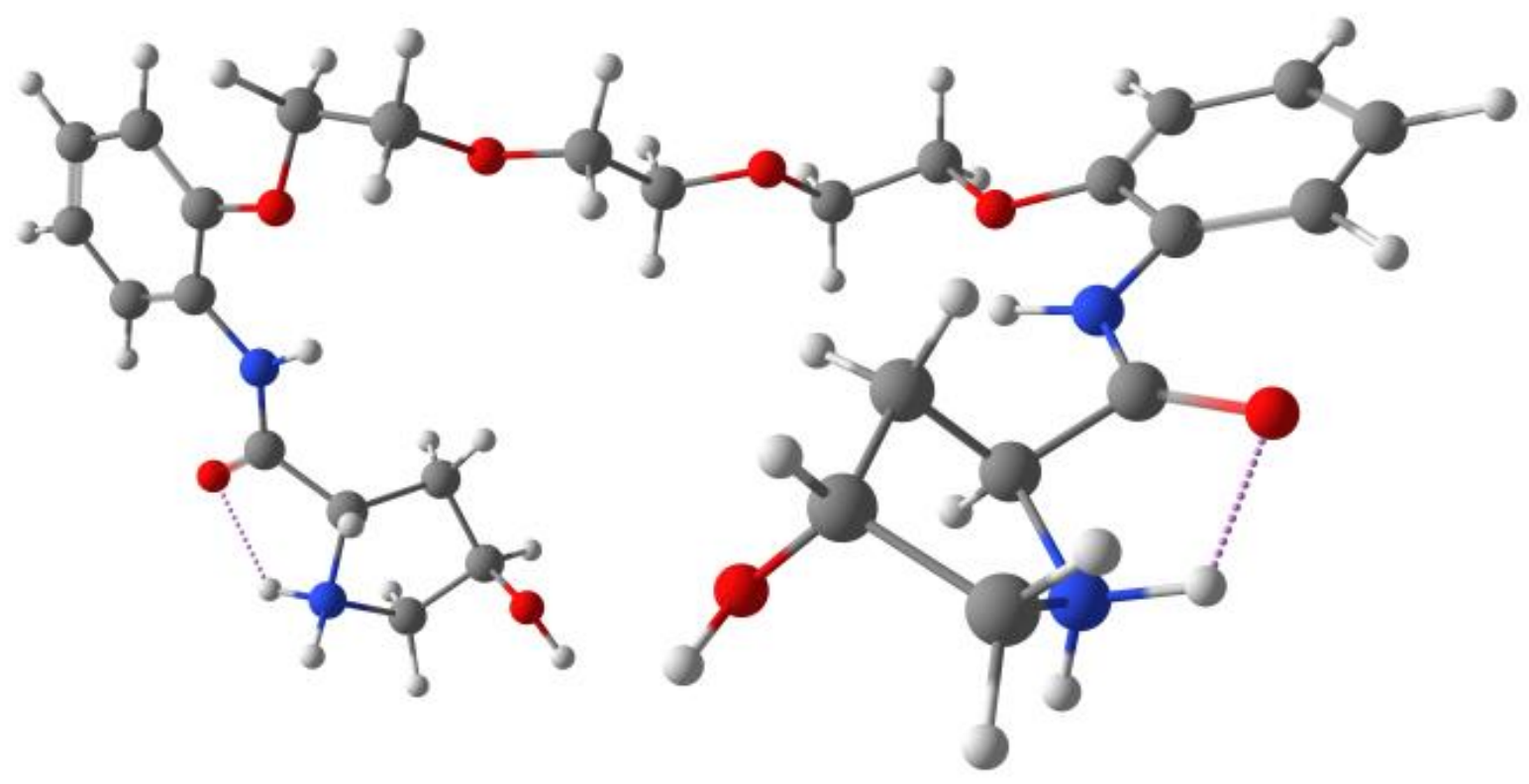

Figure S5. The final conformer of $\mathbf{2 c}(\mathrm{U} 10+171)$ taking into account the chain conformation like conformer L10-143, the arrangement of branches relative to each other like conformer Ua0+182. 

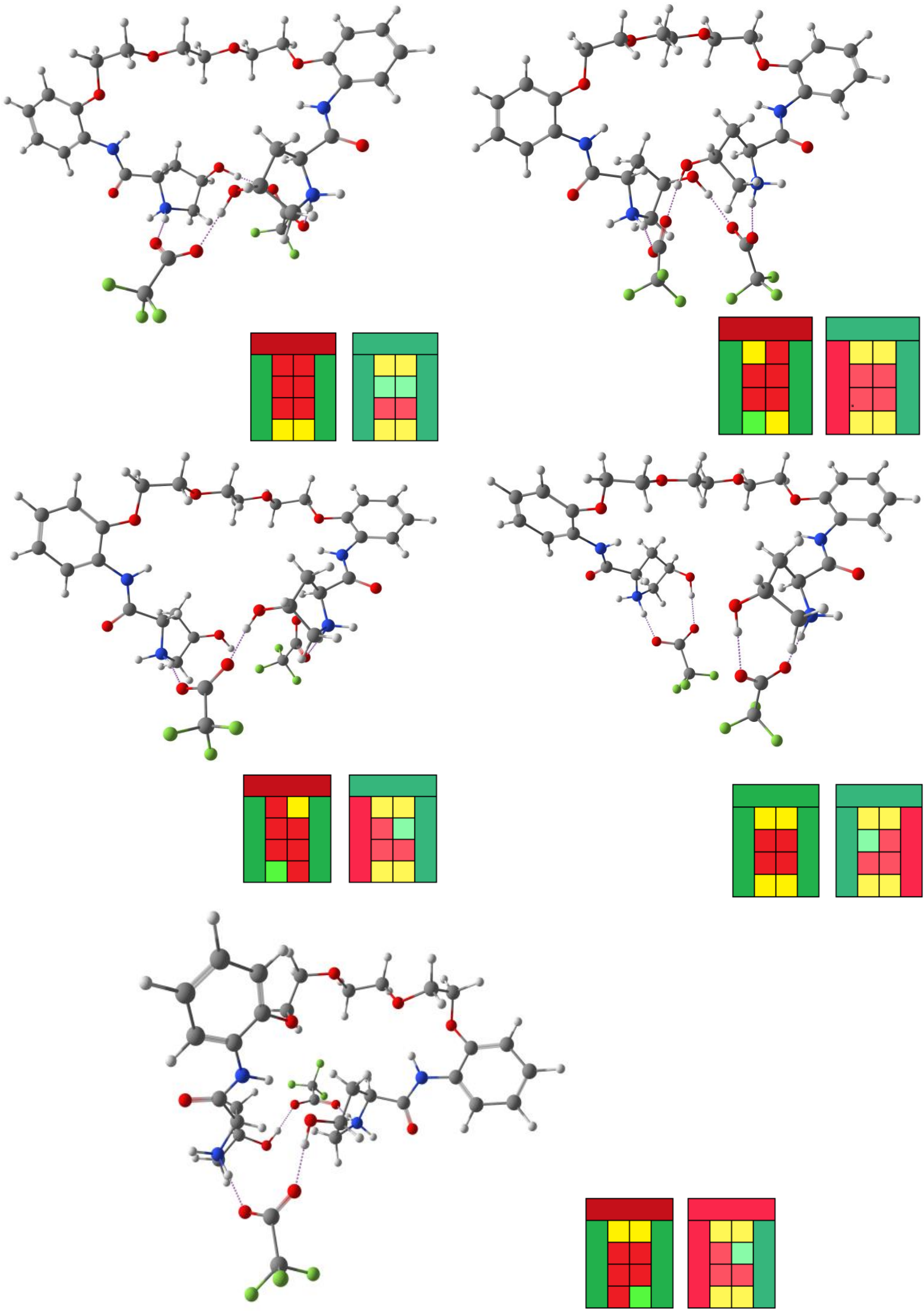

Figure S6. - Variants of trifluoroacetate anions arrangement relative to $\mathbf{2 c}$ and corresponding correlation diagrams. 